Voix et Images

volxetimages

\title{
Laure Conan à l'épreuve du livre de piété
} Hétéronomie et individuation dans la littérature québécoise du dix-neuvième siècle

\section{Laure Conan Confronted With Devotional Works:} Heteronomy And Individuation In Nineteenth-Century Quebec Literature

Laure Conan a prueba del libro piadoso. Heteronomía e individuación en la literatura quebequense del siglo XIX

\section{Marie-Andrée Beaudet}

Volume 32, numéro 3 (96), printemps 2007

Le dix-neuvième siècle québécois et ses modèles européens

URI : https://id.erudit.org/iderudit/016578ar

DOI : https://doi.org/10.7202/016578ar

Aller au sommaire du numéro

\section{Éditeur(s)}

Université du Québec à Montréal

\section{ISSN}

0318-9201 (imprimé)

1705-933X (numérique)

Découvrir la revue

Citer cet article

Beaudet, M.-A. (2007). Laure Conan à l'épreuve du livre de piété : hétéronomie et individuation dans la littérature québécoise du dix-neuvième siècle. Voix et Images, 32(3), 59-71. https://doi.org/10.7202/016578ar

\section{Résumé de l'article}

Hybrides, faisant largement appel aux genres de l'intime, les premiers textes de Laure Conan échappent aux catégories instituées du littéraire. L'hypothèse qui guide la présente relecture d'Un amour vrai (1879), "À travers les ronces " (1883) et Angéline de Montbrun (1884) est que l'oeuvre de Laure Conan s'est construite à la croisée des champs littéraire et religieux, et qu'elle doit une large part de son inspiration et de ses particularités formelles à l'influence des livres de piété français qui ont nourri l'imaginaire social de la seconde moitié du dix-neuvième siècle au Québec. L'oeuvre de Laure Conan, du moins dans ses premières manifestations, serait à prendre à la lettre, c'est-à-dire comme une contribution destinée à nourrir la ferveur des croyants et à susciter des conversions. Paradoxalement, cette hétéronomie aura favorisé l'expression d’une subjectivité indissociablement sociale et littéraire. 


\section{LAURE CONAN À L'ÉPREUVE DU LIVRE DE PIÉTÉ}

Hétéronomie et individuation dans la littérature québécoise du dix-neuvième siècle

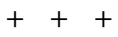

\section{MARIE-ANDRÉE BEAUDET}

Université Laval

\section{RÉSUMÉ}

Hybrides, faisant largement appel aux genres de l'intime, les premiers textes de Laure Conan échappent aux catégories instituées du littéraire. L’hypothèse qui guide la présente relecture d'Un amour vrai (1879), «À travers les ronces» (1883) et Angéline de Montbrun (1884) est que l'œuvre de Laure Conan s'est construite à la croisée des champs littéraire et religieux, et qu'elle doit une large part de son inspiration et de ses particularités formelles à l'influence des livres de piété français qui ont nourri l'imaginaire social de la seconde moitié du dixneuvième siècle au Québec. L'œuvre de Laure Conan, du moins dans ses premières manifestations, serait à prendre à la lettre, c'est-à-dire comme une contribution destinée à nourrir la ferveur des croyants et à susciter des conversions. Paradoxalement, cette hétéronomie aura favorisé l'expression d'une subjectivité indissociablement sociale et littéraire. 
$《 \ll \ll \gg \gg \gg$

Que vous le vouliez ou non, il faudra un jour être séparé de tout.

L'Imitation de Jésus-Christ

Je disais adieu au monde dans d'espèces de romances.

Arthur Rimbaud

Si l'on en croit Roland Barthes, Pierre Bourdieu et Jacques Dubois, la littérature moderne, telle que le vingtième siècle l'a connue et pratiquée, serait née en France au milieu du dix-neuvième siècle. Cette analyse fondée sur la théorie de l'autonomisation de la littérature et la séparation des marchés incite à reléguer aux limbes de la modernité littéraire à peu près toute la production canadienne-française du dixneuvième siècle. Quelles œuvres canadiennes pourraient en effet soutenir une comparaison avec Madame Bovary (1857) de Gustave Flaubert et Les fleurs du mal (1857) de Charles Baudelaire? D'où la difficulté à enseigner ce corpus qui, pour certains, relève davantage de l'histoire et de la sociologie que des valeurs esthétiques fondatrices de la modernité littéraire. Mais ne peut-on croire que cette difficulté tient pour beaucoup aux approches littéraires traditionnelles qui, en limitant l'analyse aux formes littéraires instituées, font l'impasse sur une large part de la spécificité et de l'intérêt de ce corpus hybride produit dans une société où le statut et les frontières de la littérature étaient autrement définis qu'en France, et autrement définis qu'aujourd'hui. Comme l'écrivait Robert Melançon en réfléchissant sur la notion de "classique québécois», "les classiques québécois se trouvent plus probablement dans des textes qui échappent aux catégories habituelles du littéraire que dans des poèmes et des romans sans intérêt auxquels on consacre tant de recherches par un inexplicable masochisme ${ }^{1}$ ».

Éminemment hybrides et faisant largement appel aux genres de l'intime, les premières œuvres de Laure Conan, bien que très étudiées, échappent assurément et plus qu'on a voulu le croire aux catégories instituées du littéraire. L'hypothèse qui guide la présente relecture d'Un amour vrai, "À travers les ronces» et Angéline de Montbrun ${ }^{2}$ est que l'œuvre de Laure Conan s'est construite à la croisée des champs littéraire et religieux, et qu'elle doit une large part de son inspiration et des particularités formelles de sa composition à l'influence des livres de piété français qui ont nourri l'imaginaire social de la seconde moitié du dix-neuvième siècle au Québec. L'œuvre de Laure Conan, du moins dans ses premières manifestations, serait somme

$$
++
$$

1 Robert Melançon, Qu'est-ce qu'un classique québécois?, Montréal, Fides/Les Presses de l'Université de Montréal, coll. «Les grandes conférences», 2004, p. 42-43. Melançon précise en note: « [I]l serait plus exact de dire "catégories actuelles du littéraire". Elles datent tout au plus du XIX ${ }^{e}$ siècle...» 2 Laure Conan, "Un amour vrai», Revue de Montréal, septembre 1878-août 1879, réédité sous le titre Larmes d'amour, Montréal, Leprohon, 1879; id., "À travers les ronces", Nouvelles Soirées canadiennes, vol. II, 1883, p. 340-361; id., "Angéline de Montbrun», Revue canadienne, juin 1881-août 1882, et en livre, Québec, Brousseau, 1884. Hormis pour la nouvelle «À travers les ronces» qui n'a pas connu de réédition, nous utilisons ici l'édition préparée et présentée par Roger Le Moine: Laure Conan, Euvres romanesques, vol. I (Un amour vrai et Angéline de Montbrun), Montréal, Fides, coll. «du Nénuphar», 1974. 
toute à prendre à la lettre, c'est-à-dire comme une contribution destinée - dans la tradition de L'Imitation de Jésus-Christ ${ }^{3}$ - à nourrir la ferveur des croyants et à susciter des conversions.

En définitive, l'abbé Casgrain aurait vu juste. En reconnaissant la dimension religieuse d'Angéline de Montbrun, il n'aurait fait que reconnaître ce que cette œuvre devait à la sensibilité de son époque. On sait que son célèbre commentaire

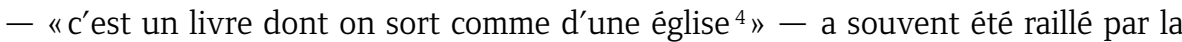
critique. Encore, tout récemment, E. D. Blodgett estimait que Casgrain avait luimême construit le contexte mystique du roman ${ }^{5}$. En outre, depuis les révélations de Roger Le Moine ${ }^{6}$, Angéline de Montbrun, loin d'être une œuvre édifiante, se voit aujourd'hui définie au contraire comme une œuvre malsaine, hantée par des fantasmes d'inceste inspirés à l'auteure par sa relation malheureuse avec un homme plus âgé, le député libéral Pierre-Louis Tremblay. Malheureusement, les preuves de cette liaison restent très minces. Elles reposent en fait sur des témoignages de seconde main, notamment sur celui d'un habitant de La Malbaie qui aurait percé le «secret» de Laure Conan. Ainsi a-t-on volontiers soutenu que Félicité Angers s'était inspirée des écrits d'Eugénie de Guérin pour exprimer, comme l'aurait fait sa célèbre devancière, un amour interdit et malheureux (l'amour démesuré pour son frère Maurice dans un cas, l'amour excessif pour un père dans l'autre), tout en négligeant de prendre en compte ce qui au dix-neuvième siècle avait fait le succès du Journal et de la correspondance de la dame du Cayla: l'exemplarité d'une aventure spirituelle. Comme le signale très justement Daniel Vaillancourt dans un récent collectif consacré à Angéline de Montbrun, « [l] a critique a, dans la majorité des cas, réduit au silence la problématique posée par la parole religieuse, voire la possibilité du religieux dans le roman ${ }^{7}$ ». Pourtant, dès 1907, le critique français Charles ab der Halden, tout en soulignant l'étroite parenté qui unit le roman de Laure Conan aux écrits d'Eugénie de Guérin ${ }^{8}$, observait que l'œuvre de la Canadienne ne débute vraiment qu'au moment où elle semble s'achever, c'est-à-dire dans la partie finale, intitulée «Feuilles détachées», qui livre le journal spirituel d'Angéline ${ }^{9}$. Il ajoutait:

$+++$

3 L'Imitation de Jésus-Christ, traduction de l'abbé Félicité de Lamennais, Paris, Nelson, 1936 [1824 pour cette traduction]. C'est l'édition que nous avons utilisée pour le présent article. Il s'agit de la traduction qui a le plus circulé au dix-neuvième siècle et que cite Laure Conan dans ses œuvres. 4 Henri-Raymond Casgrain, Euvres complètes, t. I, Montréal, Beauchemin, 1896, p. 413.5 E. D. Blogett, «Introduction», E. D. Blogett et Claudine Potvin (dir.), Relire Angéline de Montbrun au tournant du siècle, Québec, Nota bene, coll. "Convergences», 2006, p. 13. 6 Roger Le Moine, «Laure Conan et Pierre-Alexis Tremblay", Revue de l'Université d'Ottawa, vol. 36, $n^{\circ} 2$, 1966, p. 258-271; vol. 36, no 3, 1966, p. 500-528. L'auteur reprend l'essentiel de ses hypothèses dans la présentation du premier tome des Euvres romanesques de Laure Conan, op. cit., p. 9-21, où il écrit notamment (p. 18) que "tout l'échafaudage d'Angéline de Montbrun n'a d'autre fonction que de tromper le lecteur». 7 Daniel Vaillancourt, «De Laure à Marie : généalogie d'une figure», E. D. Blogett et Claudine Potvin (dir.), op. cit., p. 401. 8 Cette influence se trouve aujourd'hui bien attestée. Voir l'article de Nicole Bourbonnais, «Angéline de Montbrun, de Laure Conan : œuvre palimpseste», Voix et Images, vol. 22, n 1, 1996, p. 80-94, ainsi que la thèse de Mathilde Kang, «La fortune littéraire du Journal d'Eugénie de Guérin au Québec. Intertextualité et formes de l'intime. 1850-1950", Université du Québec à Trois-Rivières, 1998, 362 f., qui fait du cahier XI du Journal d'Eugénie de Guérin le génotexte du «Journal» d’Angéline. 9 Charles ab der Halden («Laure Conan », Nouvelles études de littérature canadienne, Paris, Rudeval, 1907, p. 196) écrit: "[Angéline] se retire à Valriant pour y pleurer son cher bonheur. Vous croyez le roman fini : Du tout, il commence.» 
«Ce dénouement est logique. Tout le roman nous y conduit ${ }^{10}$.» Aussi, l'intrigue amoureuse entre Angéline et Maurice, au lieu d'être abordée comme une transposition du drame sentimental de l'auteure, porteuse de la logique interne du roman, devrait-elle plutôt être interprétée comme un prétexte romanesque justifiant le récit de la quête spirituelle de l'hérö̈ne, la première partie offrant une sorte de portrait du monde comme il va, futile et inconstant tel que le conçoit L'Imitation de JésusChrist, et la dernière montrant le chemin par lequel il faut apprendre à le quitter.

Le fait qu'Un amour vrai, «À travers les ronces» et Angéline de Montbrun soient des œuvres sans précédent dans le panorama des lettres canadiennes de $l^{\prime}$ époque ${ }^{11}$ et que ces textes aient été écrits par la première femme écrivain au Québec ne doit pas laisser croire que ceux-ci échappent aux contraintes discursives de la société canadienne-française d'alors. Si ces romans et cette brève nouvelle s'inscrivent en effet difficilement dans la production littéraire canadienne-française du dix-neuvième siècle - plus portée vers la poésie et le roman de mœurs ou le roman historique -, ils s'inscrivent parfaitement en revanche dans le contexte du renouveau religieux mis en place dans les années 1840 par $\mathrm{M}^{\mathrm{gr}}$ Ignace Bourget. En effet, ils se font l'écho de deux fortes traditions qui connaissent alors un essor remarquable, tant en France qu'au Québec, celle des écrits à caractère religieux ou mystique - longue tradition où la présence des femmes est depuis longtemps attestée - et celle des Vies, vies de saints et de personnages héroïques, destinées à susciter ferveur et conversion, qui au Québec, contrairement à la tradition européenne, s'est largement contruite «autour du personnage féminin ${ }^{12}$ ». La publication récente de la correspondance de Laure Conan ${ }^{13}$ vient d'ailleurs appuyer fortement l'hypothèse d'une trajectoire et d'une œuvre à la croisée des champs religieux et littéraire.

\section{L'ÉCRITURE COMME VOCATION}

Si, dans sa correspondance, Félicité Angers insiste à plusieurs reprises sur la nécessité matérielle - le fait de devoir gagner sa vie après la mort de son père - qui l'aurait incitée à publier en retour d'un salaire qu'elle n'a de cesse de réclamer à ses divers correspondants du milieu littéraire, son désir d'écrire, lui, s'inscrit délibérément sous le sceau d'un appel, d'une vocation, comme elle l'affirme à sa grande amie et confidente, sœur Saint-François-Xavier de la communauté des Sœurs-du-Précieux-Sang, en 1882:

On m'encourage beaucoup à écrire. On me dit que c'est ma vocation et plusieurs choses me portent à croire qu'on dit vrai. Aidez-moi à la remplir, très chère. C'est

$$
+++
$$

10 Ibid., p. 201. 11 Gilles Marcotte (Une littérature qui se fait, Montréal, HMH, 1962, p. 16) écrit: « [Angéline de Montbrun] ne ressemble à rien de ce qui s'était écrit auparavant au Canada.» 12 Lucie Robert, «Sa vie n'est pas son œuvre. Figures féminines dans les Vies québécoises», Recherches sociographiques, vol. XLIV, n 3, 2003, p. 437.13 Laure Conan [Félicité Angers], J'ai tant de sujets de désespoir. Correspondance, 1878-1924, correspondance recueillie et annotée par Jean-Noël Dion, Montréal, Les éditions Varia, 2002. Désormais, les références à cette correspondance seront indiquées par le sigle JC. 


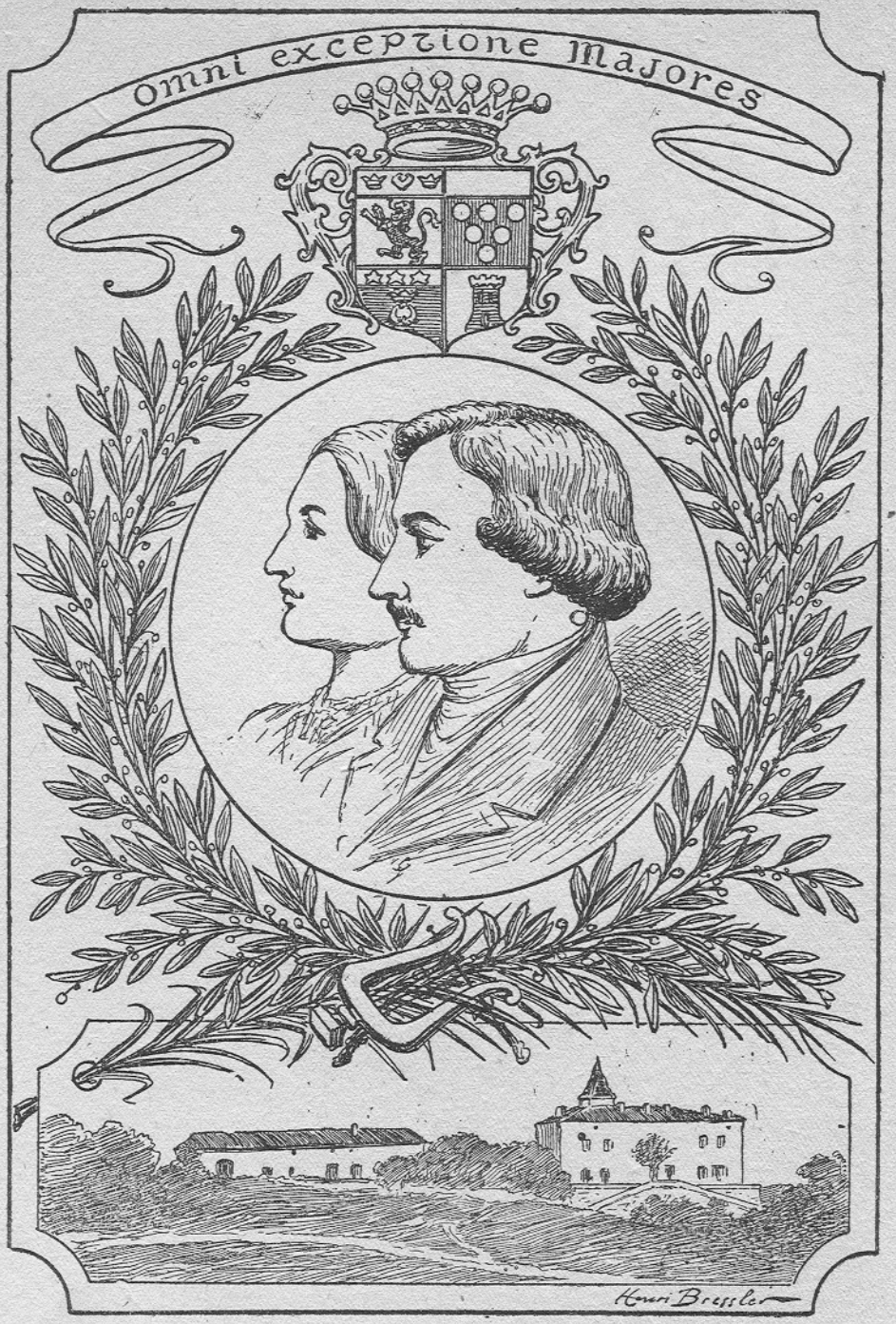

Eugénie at Maurice de Gú́rin

Armoiries des Guérin. - Ferme et château du Cayla.

Figure 1. Frontispice du Journal d'Eugénie de Guérin, représentant

Eugénie et Maurice de Guérin, les armoiries des Guérin, et la ferme et le château de Cayla. 
terrible pour une pauvre ignorante comme moi de me trouver aux prises avec une destinée pareille. Mais si Notre Seigneur voulait bénir mes faibles efforts, il peut faire fleurir les pierres et les bourbiers. C'est là-dessus que je compte. Mais encore une fois aidez-moi et faites-moi aider. Quand l'une de vos sœurs partira pour le ciel faites lui promettre de m'obtenir le succès - sans vanité ${ }^{14}$.

Il lui semble même que la sympathie que l'on témoigne à son travail littéraire «vient $\mathrm{d}^{\prime}$ en haut ${ }^{15}$ ».

Ce sentiment de répondre à un appel, Félicité le ressent depuis quelques années. Déjà au moment où elle fait ses premiers pas sur la scène littéraire et où s'amorce la publication d'Un amour vrai, elle évoque, cette fois à sœur CatherineAurélie-du-Précieux-Sang, ses projets d'écriture en termes d'action spirituelle:

J'étouffe dans cette maison où elle [sa mère qui vient de mourir] ne reviendra jamais. Il me faut absolument des occupations qui m'absorbent, qui m'arrachent à l'amertume de mes regrets. Je voudrais me mettre à écrire. Mon premier essai a été remarqué. Si c'est possible, je voudrais me servir de cette aptitude pour gagner ma vie. D'ailleurs, ces sortes d'écrits peuvent avoir aussi leur utilité. Un romancier français a publié récemment un ouvrage (Les étapes d'une conversion) qui je crois servira à faire aimer Jésus-Christ. [...] Ne pourriez-vous pas, Sweetest Mother, m'indiquer un sujet de nouvelle ou de roman? Étrange demande n'est-ce pas? Songez-y s'il vous plait. Quelque chose qui puisse faire du bien et avec la bénédiction d'en haut, faire aimer Dieu quand ce ne serait que d'une seule âme ${ }^{16}$.

Dans la perspective d'une vocation littéraire à caractère spirituel, le recours au pseudonyme de Félicité Angers doit s'entendre comme un désir de discrétion et d'humilité. Si «la publicité lui est insupportable ${ }^{17}$ » et si elle préférerait "mourir bien des fois avant d'accepter ces détails personnels ${ }^{18}$ ", comme elle le répète à l'abbé Casgrain et à différents correspondants, c'est vraisemblablement par fidélité aux préceptes de L'Imitation de Jésus-Christ, qui condamne la gloire et l'orgueil, mais peut-être également en souvenir d'Eugénie de Guérin qui, dans une lettre, avait elle-même demandé à son frère Maurice d'offrir sa pièce sur l'amitié à la Revue européenne, mais de façon anonyme: «Mais que ce soit sans nom: je ne veux être connue que de Louise [de Bayne], qui n'a pas besoin que je me nomme ${ }^{19}$.»

$14 J C$, lettre à sœur François-Xavier-du-Précieux-Sang, 28 décembre 1882, p. 140. L'italique est de Félicité Angers. $15 J C$, lettre à sœur François-Xavier-du-Précieux-Sang, 26 août 1883, p. $156.16 J C$, lettre à sœur Catherine-Aurélie-du-Précieux-Sang, 21 février 1879, p. 85-86. Le roman Les étapes d'une conversion, publié en 1878 par Paul Féval, a connu un succès fulgurant: "dix-huit éditions» l'année de sa parution, précise en note Jean-Noël Dion, le responsable de l'édition de la correspondance. Par l'expression "ces sortes d'écrits », Félicité se réfère vraisemblablement aux fictions édifiantes. $17 \mathrm{JC}$, lettres à l'abbé Casgrain, $1^{\text {er }}$ octobre 1883, p. 159160 ; 14 janvier 1884, p. 173-174; 4 mars 1884, p. 177-178. 18 JC, lettre à Thomas Chapais, 4 mars 1884, p. 179. 19 Eugénie de Guérin à son frère Maurice, 15 juillet 1834, Lettres d'Eugénie de Guérin, publiées par G. S. Trebutien, Paris, Didier et Cie, libraires-éditeurs, 1865, p. 54. 
La notion de vocation, "sommet de l'échelle des ambitions ${ }^{20}$ ", semble constitutive de la sensibilité du dix-neuvième siècle, comme le fait observer Alain Corbin. Matérialisant le désir d'individuation et d'ascension sociale qui se développe dans différentes couches de la population au dix-neuvième siècle, l'appel de la vocation migre progressivement tout au long du siècle de la sphère religieuse à la sphère profane. Si en France des ouvriers entendent l'appel républicain, il semble qu'au Québec, le phénomène soit demeuré principalement lié au domaine religieux, encore que le terme s'utilisait également, mais avec des connotations atténuées, pour le mariage alors considéré comme un sacrement. À cet égard, la vocation littéraire de Félicité Angers semble donc le signe d'une mutation sociale. Ne ressentant aucun attrait, comme elle l'écrit, ni pour la vie cloîtrée ni pour le mariage ${ }^{21}$, elle trouve dans l'adoption d'une vocation littéraire à caractère spirituel un compromis à la fois acceptable pour son époque et conforme à ses croyances.

«Être utile», «faire du bien», tels sont les maître-mots et les ambitions qui animent Laure Conan, dans ses projets d'écriture comme dans ce grand projet de création d'un nouveau monastère pour les religieuses du Précieux-Sang qu'elle entrevoit à Chicoutimi. Dans la réalisation de sa vocation d'écrivain, ses mentors sont moins les acteurs du milieu littéraire que les prêtres et les religieuses avec lesquels Félicité est en correspondance suivie: au premier titre, sœur Catherine-Aurélie, fondatrice des Sœurs-du-Précieux-Sang, première communauté contemplative à voir le jour au Québec avec l'appui de $M^{\mathrm{gr}}$ Bourget, sœur François-Xavier, le père Louis Fievez, l'abbé Paul Bruchési, le père Édouard Désy, etc. C'est à eux qu'elle demande prières et encouragements pour le succès de son entreprise. Si Félicité sollicite à l'occasion quelques hommes de lettres influents (l'abbé Casgrain, Pierre Chauveau, Alfred Garneau, Thomas Chapais), il reste qu'elle s'ouvre davantage à ses conseillers spirituels à propos de ses projets d'écriture: «J'aurais envie d'écrire une toute petite chose qui viendrait à dire qu'avec J. C. c'est la tristesse qui est un songe et la joie qui est une réalité. Quelques pages là-dessus adressées aux Canadiennes. Qu'en pensezvous? M'aiderez-vous ${ }^{22}$ ?» Ou encore : «M. [l'abbé] Bruchési me conseille aussi très fortement de la faire imprimer [Angéline de Montbrun]. C'est à lui que j'ai communiqué mon dernier travail auquel il a donné l'imprimatur ${ }^{23}$.»

Ses correspondants répondent volontiers à ses appels en intervenant en sa faveur auprès des directeurs de périodiques. Ainsi l'abbé Bruchési, celui que Félicité désigne comme un "ange de prêtre ${ }^{24}$ ", fait part à Thomas Chapais de son appréciation du manuscrit $\mathrm{d}^{\prime}$ «A $\mathrm{A}$ travers les ronces» en ces termes: «style riche, pensées élevées, sentiments admirables ${ }^{25}$ ", en le priant d'intervenir auprès de Louis-Hyppolite Taché pour la publication du texte dans les Soirées. Le même abbé Bruchési fera

$$
++
$$

20 Alain Corbin, "Le secret de l'invididu», Philippe Aries et Georges Duby (dir.), Histoire de la vie privée, vol. IV. De la Révolution à la Grande Guerre, Michelle Perrot (dir.), Paris, Éditions du Seuil, coll. "Points Histoire», 1999, p. 430. 21 Félicité (JC, lettre à sœur François-Xavier-du-Précieux-Sang, 11 novembre 1884, p. 186) écrit: «Je n'ai pas l'ombre d'une inclination pour le cloître - également pas la moindre pour le mariage - ce grand sacrement ne m'attire pas... Ah que j'y souffrirais!» $22 \mathrm{JC}$, lettre à sœur FrançoisXavier-du-Précieux-Sang, 30 novembre 1884, p. 187. 23 JC, lettre à l'abbé Casgrain, 15 août 1883, p. 154 24 Ibid., p. 152.25 Ibid. 


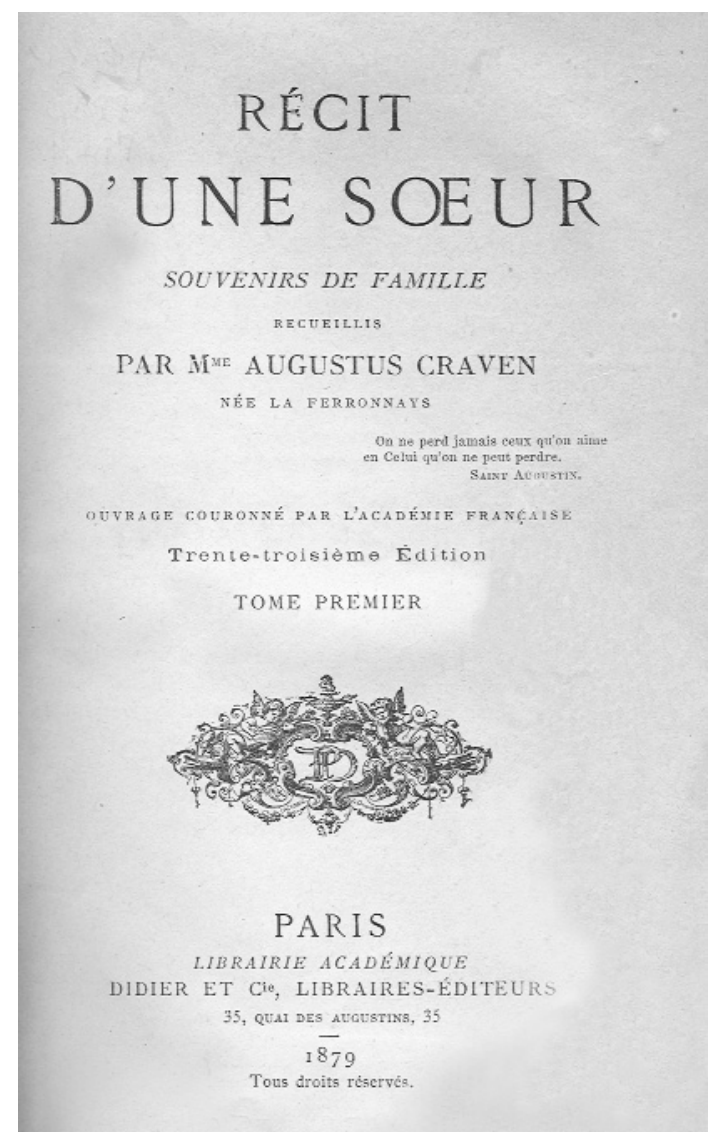

Figure 2. Page de titre de Récit d'une sœur. Souvenirs de famille,

recueillis par $\mathrm{M}^{\mathrm{me}}$ Augustus Craven, Paris, Didier et Cie, libraires-éditeurs, 1879.

connaître l'œuvre de Laure Conan à Madame Craven, l'auteure de Récit d'une sœur. Souvenirs de famille ${ }^{26}$, que Félicité admire et avec laquelle elle partage comme avec Eugénie de Guérin et ses autres auteurs de prédilection une passion pour L'Imitation de Jésus-Christ et une détestation du siècle.

\section{EN EXIL DANS LE SIÈCLE}

Il est étonnant que l'on ne se soit guère intéressé jusqu'ici à la dimension politique de l'œuvre de Laure Conan qui, bien qu'intimement liée à ses croyances religieuses, permet d'éclairer la posture à la fois mélancolique et volontariste qui caractérise

$$
+++
$$

$26 \mathrm{M}^{\mathrm{me}}$ Augustus Craven, Récit d'une sœur. Souvenirs de famille (recueillis par), t. I, Paris, Didier et Cie, libraireséditeurs, 1879. 
l'écriture de la romancière. Ses premières œuvres et sa correspondance offrent pourtant bien des indices à cet égard. Dans Angéline de Montbrun, Charles de Montbrun et sa fille, présentés comme des êtres d'exception, affichent clairement leurs positions anti-républicaines à l'encontre des convictions (silencieuses) du pauvre Maurice, décrit comme un être faible et sentimental, associé au monde de l'inconstance et des plaisirs éphémères :

C'est aujourd'hui la Saint-Louis. Nous ne l'avons pas oublié. Pauvre France! Angéline dit, comme Eugénie de Guérin, qu'elle filerait volontiers la corde pour pendre la République et les républicains. [...] Angéline est plus royaliste que moi: elle me trouve tiède, et Maurice n'ose dire qu'il est bonapartiste ${ }^{27}$.

Si les Montbrun fuient le siècle et les bruits du monde en célébrant les grandeurs oubliées de l'Antiquité et du Moyen Âge, Maurice paraît au contraire incarner l'attachement coupable aux réalités du monde contemporain. Ainsi, Charles de Montbrun, en lui reprochant de "se mêler d'élections» et de faire "de beaux discours sur la conscience», acceptera-t-il de lui donner sa fille en mariage à la condition qu'il abandonne son intérêt pour la politique : « Nous avons eu nos grandes luttes parlementaires. Mais c'est maintenant le temps des petites: l'esprit de parti a remplacé l'esprit national. [...] Que ce qui est grand reste grand, que ce qui est pur reste pur ${ }^{28}$.»

Dans sa correspondance, Félicité Angers soutient des positions semblables. La situation de la France républicaine la désespère. Comme ses confidentes de la communauté des Sœurs-du-Précieux-Sang, elle s'inquiète du sort des Bourbons et prie pour les Français "qui auraient grand besoin d'être réchauffés ${ }^{29}$ ». Sœur CatherineAurélie lui écrit: " [N]e pensez-vous pas, amie, qu'il faudra encore du Sang versé pour régénérer la France, si toutefois Dieu ne l'a pas encore rejetée ${ }^{30}$. » À la nouvelle de la mort du comte de Chambord, Félicité fait part de son désarroi à sœur FrançoisXavier: «Pauvre, pauvre France! Comment saint Louis a-t-il pu le laisser mourir... Je suis consternée ${ }^{31}$.»

Comme les auteurs qu'elle fréquente, Félicité Angers éprouve le sentiment de vivre en exil dans un "siècle d'énervement et de faiblesse ${ }^{32}$ ». Elle déteste le «terne» et le « tiède» qui affligent l'existence de ses contemporains. Ses aspirations la portent vers les êtres forts, ces saints et ces héros dont on publie alors les Vies et qui, contrairement à l'historien François-Xavier Garneau qu'elle admire, sont pour l'essentiel des figures du passé. En regard, le présent paraît privé de consistance. Ce qu'exprime également Angéline de Montbrun: «Je ne vis guère dans le présent, et pour ne pas voir l'avenir, qui m'apparaît comme une morne et désolée solitude, je

$$
+++
$$

27 Laure Conan, "Lettre de Mina à Emma», Angéline de Montbrun, op. cit., p. 138. L'italique est de Conan. 28 Ibid., «Lettre de Charles de Montbrun à Maurice», p. 118-119. 29 JC, lettre à l'abbé Casgrain, p. 149. $30 J C$, lettre de sœur Catherine-Aurélie-du-Précieux-Sang à Laure Conan, 10 octobre 1882, p. 137. 31 JC, lettre à sœur François-Xavier-du-Précieux-Sang, 26 août 1883, p. $156.32 J C$, lettre à sœur Catherine-Auréliedu-Précieux-Sang, 20 octobre 1879, p. 102. 
songe au passé tout entier disparu. Ainsi le naufragé, qui n'a que l'espace devant lui, se retourne, et dans sa mortelle détresse, interroge la mer où ne flotte plus une épave ${ }^{33}$.» Félicité Angers souffre ne pas être née dans le bon siècle, comme Mina qui écrivait à son frère: "Pour moi, j'ai toujours regretté de n'être pas née dans les premiers temps de la colonie, alors que chaque Canadien était un héros ${ }^{34}$.»

Si ce rapport problématique au présent renvoie de façon évidente à l'idéologie ultramontaine et participe de cette opposition marquée "entre l'esprit du temps et la foi traditionnelle» qui caractérise le dix-neuvième siècle, comme le signale René Rémond ${ }^{35}$, il trahit également par l'accent mis sur les destins d'exception ce puissant désir d'individuation qui touche alors tous les milieux sociaux et qui permettra aux femmes d'accéder à une plus grande autonomie. L'intérêt marqué pour la pratique du journal intime et de la correspondance - formes dominantes des premières œuvres de Laure Conan - témoigne également de cette volonté nouvelle d'exprimer les ambitions du moi et d'explorer ne serait-ce que par écrit les conditions de sa réalisation. Dans cette perspective, le précepte de L'Imitation qui veut qu'il ne faille «tenir à rien au dehors ${ }^{36}$ » ne pouvait s'entendre pour Laure Conan sans contradictions ni souffrances.

\section{DOLORISME ET TRANSPORTS MYSTIQUES}

La lecture comparée des premières œuvres de Laure Conan, de sa correspondance et des ouvrages religieux auxquels elle se réfère, montre à l'évidence l'importante circulation à la fois intime et sociale des thèmes et du vocabulaire mystiques. Il faut rappeler que l'imagerie gothique, magnifiant les souffrances du Christ, connaît une grande vogue dans les milieux catholiques de la seconde moitié du siècle. Cette sensibilité nouvelle, qui culmine en France sous la Restauration, sera diffusée au Canada par certains ouvrages romantiques, mais surtout, et de façon plus décisive, par les nombreuses communautés religieuses ${ }^{37}$ que $\mathrm{M}^{\mathrm{gr}}$ Bourget fait venir au pays aux lendemains des Insurrections pour tenter de raviver une pratique qui déclinait et consolider l'autorité du pape et du clergé dans tous les secteurs de l'activité sociale. Avec l'appui des communautés venues de France, $\mathrm{M}^{\mathrm{gr}}$ Bourget misera sur une religion populaire qui n'hésite pas à recourir au pathos et aux procédés spectacu-

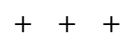

33 Laure Conan, Angéline de Montbrun, op. cit., p. 182. 34 Ibid., "Lettre de Mina Darville à son frère», p. 108. 35 René Rémond, Introduction à l'histoire de notre temps, t. II. Le XIXe siècle. 1815-1914, Paris, Éditions du Seuil, coll. «Points Histoire», 1974, p. 200. 36 L'Imitation de Jésus-Christ, op. cit., p. 106. 37 Outre ces communautés venues de France, plusieurs nouvelles communautés sont fondées sous l'impulsion de $\mathrm{M}^{\mathrm{gr}}$ Bourget (Nive Voisine, Histoire du catholicisme québécois, t. II. 1840-1898 [par Philippe Sylvain et Nive Voisine], Montréal, Boréal, 1991, p. 275-276): «En passant, de 1850 à 1901, de 361 à 2391 membres - dont 1984 œuvrant au Québec - les communautés religieuses d'hommes connaissent un progrès rapide. Mais pour les congrégations féminines, il faut parler d'une véritable floraison: de 673 à 9601 religieuses! De ce nombre, 6628 travaillent au Québec dans les domaines les plus variés. Elles se répartissent dans 36 communautés diverses, dont 15 nouvelles implantées entre 1871 et 1899: sept venues de l'étranger, huit fondées sur place», dont la communauté des Sœurs-du Précieux-Sang avec laquelle Laure Conan entretient des relations privilégiées allant jusqu'à diriger leur revue, La Voix du Précieux-Sang, de 1894 à 1898. 
laires (édifications de croix, longues retraites paroissiales, prédications flamboyantes, chants, processions, etc.) afin de toucher les cœurs et susciter la crainte de Dieu. Apparaissent alors, sous l'influence conjuguée du romantisme et de l'ultramontanisme, de nouveaux rituels et une nouvelle rhétorique qui font appel à l'émotion et à une imagerie sanglante proche du gothique. Comme le dit Alain Corbin:

Dans cette littérature, qui inspire l'école néolamartinienne, le sang coule, jaillit, recouvre le corps du Crucifié. L'usage impose de ceindre le Cœur de la couronne d'épines. [...] La dévotion à Marie, elle aussi, témoigne de cette fascination; NotreDame des Sept-Douleurs, la figure du Stabat mater focalisent la piété mariale. [...] La pratique reflète cette sensibilité tragique, confortée par la croyance en la circulation du sang du Christ dans l'histoire ${ }^{38}$.

L'œuvre de Laure Conan s'avère toute imprégnée de cette nouvelle sensibilité et le journal d'Angéline de Montbrun offre un véritable inventaire des pratiques qui lui sont associées: images saintes - celle de Notre-Dame des douleurs «que quatre épingles fixaient sur le mur ${ }^{39}$ » au pied du lit de mourant de Charles de Montbrun; prémonitions - celle que ressent Angéline la veille de la mort de son père et celle qui préfigure l'accident funeste qui la privera de sa beauté ${ }^{40}$; visions - dans un étrange songe, Angéline voit Véronique Désileux « au fond de sa fosse ${ }^{41}$ » et lors d'un grand abattement il lui semble entendre la voix du chrétien qui «du fond de sa tombe, chantait ses immortelles tendresses et ses impérissables espérances ${ }^{42}$ "; dévotions à la Vierge; série de scènes d'agonie accompagnées de pâleurs et de frayeurs, etc. En outre, par le biais de micro-histoires (celles de Véronique Désileux, de Marie Desroches et de cette pauvre veuve de marin «restée sans autre ressource que son courage, pour élever ses trois enfants ${ }^{43}$ »), le journal sert également à illustrer l'un des enseignements de L'Imitation qui préconise la fréquentation des gens pauvres et simples en raison de la plus grande pureté de leur foi ${ }^{44}$.

Les lettres que Félicité Angers échange avec sœur Catherine-Auréliedu-Précieux-Sang, et qui s'ouvrent le plus souvent sur un «Vive le sang de Jésus!», sont encore plus éloquentes à cet égard. On y retrouve, surtout sous la plume de la fondatrice des Sœurs-du-Précieux-Sang, une profusion de termes et de métaphores associées tant à l'imaginaire gothique qu'au vocabulaire mystique:

Je viens vous saluer dans les Plaies de Jésus - ressuscité - et vous souhaiter aujourd'hui la visite d'un Ange qui vous fasse connaître et accomplir la volonté divine. [...] Ne craignez pas de marcher sur ses traces sanglantes, ma pieuse Amie, car plus vous serez crucifiée maintenant, plus vous serez glorifiée un jour [... ${ }^{45}$.

$$
+++
$$

38 Alain Corbin, op. cit., p. 441-442. 39 Laure Conan, Angéline de Montbrun, op. cit., p. 161.40 Ibid., p. 195.41 Ibid., p. 175.42 Ibid., p. 199. 43 Ibid., p. 191.44 "Recherchez les humbles, les simples, les personnes de piété et de bonnes mœurs; et ne vous entretenez que de choses édifiantes. " (L'Imitation de Jésus-Christ, op. cit., p. 38.) 45 JC, lettre de sœur Catherine-Aurélie-du-Précieux-Sang à Laure Conan, avril 1880, p. 112-113. 
Dans le Sang dont je couvre bien souvent votre âme, je suis toujours avec dévouement, reconnaissance et affection, Votre bien attachée $[\ldots]^{46}$.

Que Jésus crucifié vous montre ses blessures et vous enivre de son Sang ${ }^{47}$.

Que Marie Immaculée vous introduise dans la blessure bénie du Cœur de Jésus; là, ma chère et pieuse amie, enivrez-vous de Sang, embrasez-vous d'amour, imprégnezvous de sainteté ${ }^{48}$.

L'influence de cette rhétorique doloriste sur les premières œuvres de Laure Conan est manifeste. À défaut de pouvoir en fournir un relevé complet, nous nous contenterons de deux exemples, le premier tiré d'Un amour vrai : "Quand je regardai [le crucifix] là à côté de Thérèse morte, ce fut comme si une lumière éclatante jaillissant des plaies sacrées du Christ eût illuminé les mystérieuses profondeurs de l'éternité ${ }^{49}$.» Le second exemple est un extrait d'Angéline de Montbrun, plus précisément des dernières lettres échangées entre Maurice et Angéline qui s'offrent comme l'ultime morale à l'histoire :

[...] comme disait Mina Le sang du Christ vous unira. Chrétienne, avez-vous compris la force et la suavité de cette union? Doutez-vous que dans son sang nous ne trouvions l'immortalité de l'amour, les joies profondes du mutuel pardon. [...] Mais il en est qui n'arrivent au ciel qu'ensanglantés, et ceux-là n'ont pas droit de se plaindre ${ }^{50}$.

\section{DE L'INFLUENCE DE L'IMITATION ET DES LIVRES DE PIÉTÉ}

Dans une époque où la mort s'avère une présence familière (les nombreuses morts qui parsèment Un amour vrai et Angéline de Montbrun sont éloquentes à cet égard), les méditations sur les épreuves et les fins dernières sont particulièrement prisées. Les livres de piété, destinés à apaiser et à guider les âmes troublées, connaissent une vogue sans précédent. Témoignages de convertis, vies exemplaires, revues, annales envahissent, sous le patronage de L'Euvre des bons livres, fondée en 1845, les bibliothèques paroissiales et les cabinets de lecture. À ce propos, dans l'évaluation des réalités de la lecture au dix-neuvième siècle, il convient, comme le souligne Alain Corbin, «de ne pas se laisser aveugler par les prestiges de l'histoire littéraire ${ }^{51}$ ». Si les travaux de Claude Savard ${ }^{52}$ ont montré pour la France quelle était, en 1861, l'ampleur de la diffusion de la littérature de piété, les études statistiques menées par Yvan Lamonde et Sophie Montreuil à partir des collections de Bibliothèque et

$$
+++
$$

46 JC, lettre de sœur Catherine-Aurélie-du-Précieux-Sang à Laure Conan, 23 novembre 1880, p. 117.47 JC, lettre de sœur Catherine-Aurélie-du-Précieux-Sang à Laure Conan, 24 mars 1881, p. 123.48 JC, lettre de

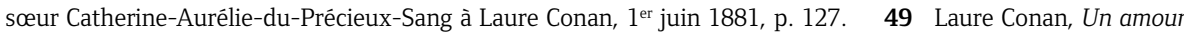
vrai, op. cit., p. 63. 50 Id., "Lettre de Maurice», Angéline de Montbrun, op. cit., p. 238; ibid., "Lettre d'Angéline», p. 240. 51 Alain Corbin, op. cit., p. 455. 52 Claude Savard, "Le livre catholique témoin de la conscience religieuse en France au XIX siècle», thèse de doctorat, Paris-Sorbonne, 1981. 

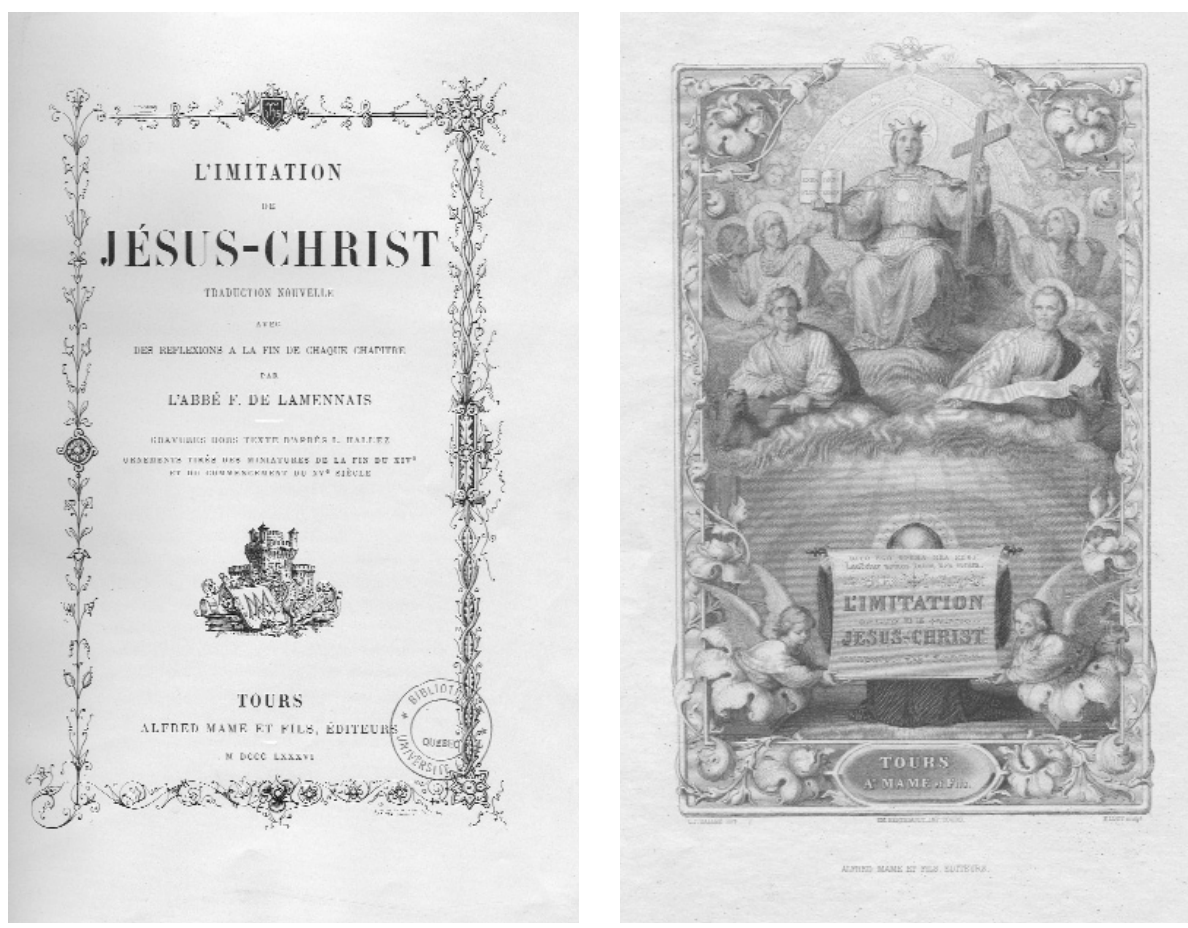

Figures 3 et 4. Page de titre et frontispice de L'Imitation de Jésus-Christ,

traduction de l'abbé Félicité de Lamennais, Tours, Alfred Mame et fils éditeurs, 1886.

Archives nationales du Québec révèlent qu'entre 1840 et 1918, le livre religieux domine nettement les autres catégories d'ouvrages imprimés au pays ${ }^{53}$.

De tous les livres de piété, le plus lu et le plus médité au dix-neuvième siècle et jusque tard au vingtième reste L'Imitation de Jésus-Christ, œuvre du quinzième siècle traduite par l'abbé Félicité de Lamennais en 1824, dont Eugénie de Guérin vante les mérites dans sa correspondance: "L'admirable ouvrage! Quelle connaissance du Cœur! Il y a des passages pour toutes les situations de l'âme, des remèdes pour chaque passion, une mansuétude divine ${ }^{54}$.» Au Canada, ce guide spirituel connaît également une grande popularité comme l'illustre ce passage du Journal de Joséphine Marchand: «Hier dimanche, écrit-elle dans son Journal du $1^{\text {er }}$ mars 1886, nous avons délicieusement flâné toute la journée, après avoir été à la messe comme de bons chrétiens et avoir lu (c'est Raoul qui m'a fait la lecture) deux chapitres de lecture spirituelle de L'Imitation de Jésus-Christ ${ }^{55}$."

53 Joséphine Marchand, Journal, cité dans Yvan Lamonde et Sophie Montreuil, «Aperçus quantitatifs rétrospectifs de l'imprimé au Québec», Yvan Lamonde, Patricia Fleming et Fiona A. Black (dir.), Histoire du livre et de l'imprimé au Canada, t. II. De 1840 à 1918, Montréal, Les Presses de l’Université de Montréal, 2005, p. 521. 54 Lettre d'Eugénie de Guérin à Madame la baronne de Maistre, 4 mai 1838, Lettres d'Eugénie de Guérin, op. cit., p. 164. 55 Sophie Montreuil, "(Se) lire et (se) dire : Joséphine Marchand et la lecture (1879-1886) », Yvan Lamonde et Sophie Montreuil (dir.), Lire au Québec au XIXe siècle, Montréal, Fides, 2003, p. 137. 
La morale très exigeante de L'Imitation, qui prône le détachement de soi et le renoncement au monde, convenait bien à ceux et à celles qui, comme Félicité Angers, ne trouvaient autour d'eux et dans le siècle qu'indifférence et tiédeur. Le caractère à la fois intense et mesuré de la prose de L'Imitation, qui dispose à la citation comme à la méditation, explique en grande partie sa popularité ${ }^{56}$. Les journaux intimes dont Laure Conan fait un grand usage dans ses fictions font tous référence à L'Imitation. Ainsi, «Ā travers les ronces», journal spirituel d'une âme tiède - celle de Valérie B. qu'une narratrice offre à la réflexion d'une correspondante fictive, «Madame S.S. » - met en scène un moment de méditation inspiré par la lecture de L'Imitation:

\author{
L'Imitation dit: C'est faire beaucoup que d'aimer beaucoup. C'est faire beaucoup que \\ de faire bien ce que l'on fait. \\ Il y a dans cette parole la condamnation de bien des rêves et des regrets. \\ Qu'importe à nous-mêmes et aux autres, l'éclat de nos œuvres? \\ Se trouver mal placée, mal partagée, n'est-ce pas dire à Dieu: Je sais mieux que \\ vous ce qui me convient. Grande audace, ma pauvre Valérie ${ }^{57}$.
}

Dans Angéline de Montbrun, Véronique Désileux, qui agit comme un adjuvant important dans la quête spirituelle d'Angéline, cite un précepte de L'Imitation dans la lettre posthume qu'elle lui adresse: «Tel est votre bien-aimé, qu'il ne veut point de partage; il veut posséder seul votre Cœur, et y régner comme un roi sur le trône qui est à lui ${ }^{58}$.» Cet extrait du deuxième livre de L'Imitation, intitulé «Instruction pour avancer dans la vie intérieure », énonce en fait le parcours proto-mystique suivi par Angéline dans le roman: des plaisirs du monde, que dessine en première partie l'échange de correspondances, au dur apprentissage de la solitude et du détachement auquel Dieu convie le croyant par les épreuves, afin d'accéder au pur Amour: «Il est bon d'avoir quelquefois des peines et des traverses, parce que souvent elles rappellent l'homme à son Cœur, et lui font sentir qu'il est en exil, et qu'il ne doit mettre son espérance en aucune chose du monde ${ }^{59}$."

Outre L'Imitation et Eugénie de Guérin, plusieurs autres auteurs de livres religieux ou édifiants sont cités ou évoqués dans Angéline de Montbrun, dont Madame Swetchine, Madame Craven, Saint-Augustin, Lacordaire, Ignace de Loyola. On y évoque aussi la lecture de Tout pour Jésus et du Vol de l'âme dont les titres seuls, à défaut de pouvoir identifier leurs auteurs, signalent la teneur spirituelle. La majorité de ces ouvrages offre la particularité d'être des essais ou des témoignages vécus, livrés par le biais de correspondances ou de journaux intimes (en accueillant parfois les deux formes à la fois) dont les auteurs ou les éditeurs sont très souvent des femmes. Comme le dit Alain Corbin, « [l]'essor du journal féminin de conversion au XIX siècle traduit la [...] volonté d'adapter le besoin croissant d'écriture de soi à

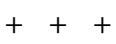

56 Dans l'une de ses chroniques, Gilles Marcotte ("Au lecteur absent», Liberté, n 227, 1996, p. 46) rappelait que Saint-Denys Garneau comparait L'art de la fugue de Jean-Sébastien Bach à L'Imitation de Jésus-Christ. 57 Laure Conan, "À travers les ronces», op. cit., p. 350.58 Id., Angéline de Montbrun, op. cit., p. 174.

59 L'Imitation de Jésus-Christ, op. cit., p. 46. 
des fins édifiantes ${ }^{60}$ ». Si l'œuvre de Laure Conan offre manifestement une étroite parenté de ton et de forme avec ces récits vrais, elle s'en distingue pourtant par sa dimension fictionnelle.

\section{L'USAGE DU ROMAN}

Connaissant l'aversion des milieux catholiques du dix-neuvième siècle pour le roman, on peut s'étonner du choix de Laure Conan. En France comme au Québec, en effet, le genre romanesque fait partie de ces mauvaises lectures dont on dénonce les "effets ravageurs ${ }^{61}$ ». Partageant les craintes de son époque, Eugénie de Guérin met en garde l'une de ses correspondantes: «Les romans me font l'impression de la poudre : ils brûlent, noircissent, déchirent le cœur; les bonnes lectures l'éclairent, le fortifient, le nourrissent. Les bons livres, c'est la manne du peuple de Dieu, la céleste nourriture des âmes pour le voyage du ciel; recueillons-la ${ }^{62}$."

Genre mondain, associé à la frivolité et à la corruption, le roman est surtout considéré comme dangereux pour les jeunes filles et les âmes sensibles. Aussi Laure Conan l'aborde-t-elle avec d'infinies précautions. À l'entrée de "À travers les ronces", sa première publication, elle prévient qu' «il ne faut pas s'attendre à ce qui fait le charme du roman ${ }^{63}$ » et elle ajoute en conclusion de sa nouvelle - qu'elle définit comme un «entretien d'une âme avec elle-même» - que «la réalité n'est pas le roman, et [que] l'important dans la vie, c'est de se résigner à l'insuffisant, au vide, au froid, au terne ${ }^{64} »$. Laure Conan fait aussi rapidement préciser par Mina Darville que la très pure Angéline a été préservée de sa fréquentation: «[N]'ayant jamais lu de romans, elle [Angéline], ne s'inquiète pas des larmes que la pénétrante douceur de ton chant lui fait verser ${ }^{65}$."

Dans sa correspondance, l'auteure cherche également à se justifier de l'adoption de ce genre honni:

[...] je vais commencer un roman. Je me sers de ce mot quoiqu'il ne rende pas bien ma pensée. En effet, il s'agit seulement de l'histoire intime, l'histoire d'une âme si vous voulez. Je voudrais montrer une âme passionnée luttant contre non une grande douleur mais contre le terne. Si je réussissais, ce livre ferait un grand bien je ne doute pas, car le terne voilà l'ennemi, voilà ce qui fait le fond de la vie ${ }^{66}$.

En mettant sa vocation d'écrivain au service du bien, tel que le conçoit l'idéologie ultramontaine, Laure Conan aspire en fait à imprimer à ses productions romanesques les visées de l'imprimé religieux, qui peut être défini, selon Louis Rousseau, "par l'intention centrale autour de laquelle s'articulent ses diverses formes, qui consiste à

60 Alain Corbin, op. cit., p. 422.61 Ibid., p. 164.62 Lettre d’Eugénie de Guérin à la baronne de Maistre, 4 mai 1838, Lettres d’Eugénie de Guérin, op. cit., p. 164.63 Laure Conan, "À travers les ronces», op. cit., p. 341. 64 Ibid., p. 361.65 Id., «Lettre de Mina Darville à son frère Maurice», Angéline de Montbrun, op. cit., p. 98.66 JC, lettre à sœur Saint-François-Xavier-du-Précieux-Sang, 28 décembre 1882, p. 140. 
encourager le lecteur à développer des croyances et des conduites qui lui permettront d'accéder aux "biens de salut", garants de la réussite ultime de l'existence humaine ${ }^{67}$ ». Cet usage particulier du roman, ce détournement du genre au profit du livre religieux se heurtera cependant, particulièrement dans Angéline de Montbrun, à quelques obstacles. Si, de toute évidence, Laure Conan, comme son hérö̈ne, est tentée par l'aventure mystique, ses attaches au monde restent très fortes. Le corps résiste. Dans cette résistance, quelque chose d'autre, quelque chose d'étranger au projet initial se fait jour. Si le monde semble progressivement s'éloigner et disparaître dans la dernière partie du roman au profit de l'espace intérieur, ce retrait n'est pas sans éclairer à la fois le fort sentiment d'exil que ressent Laure Conan à l'égard de son siècle et les conditions d'exercice de sa vocation d'écrivain.

En effet, si sa correspondance et ses premières œuvres romanesques témoignent d'une grande fascination pour l'univers et le langage mystiques incarnés par le culte de ses amies et confidentes, les Sœurs-du-Précieux-Sang, il demeure que c'est dans l'espace littéraire et l'usage du roman que Laure Conan trouvera le lieu d'habitation de ce langage et des transports qui lui sont attachés.

Paradoxalement, l'originalité de son œuvre réside dans cet étonnant mélange de discours et d'influences formelles que ses fictions romanesques intègrent et interrogent. Si cette hybridité discursive et générique explique en bonne part l'effet d'étrangeté que produit Angéline de Montbrun sur les lecteurs d'aujourd'hui, elle souligne également le faible statut de la littérature québécoise au dix-neuvième siècle et permet d'illustrer l'observation déjà faite par Lucie Robert: " [S]i le champ littéraire [canadien-français du dix-neuvième siècle] est hétéronome, les pratiques d'écriture qui le traversent doivent l'être tout autant et marquer, dans leur texture même, l'entrecroisement des diverses valeurs qui le fondent ${ }^{68}$." Au nombre de ces valeurs qui ont affecté tant l'autonomie de la littérature québécoise au dix-neuvième siècle que la conformité aux règles et genres prescrits par la France, il paraît essentiel d'inclure les valeurs religieuses, en ne sous-estimant pas toutefois le pouvoir de subversion que recelaient, en dépit de leur conservatisme, ces nouvelles formes de dévotion axées sur la lecture et l'intériorité.

En définitive, l'examen des premières œuvres de Laure Conan montre que si la littérature canadienne-française du dix-neuvième siècle a de toute évidence subi l'influence du canon français, elle s'est aussi inventée pour une bonne part à travers la réception de textes qui échappaient aux modèles littéraires institués. Dans ses effets de marge, cette double hétéronomie, à la fois interne et externe, n'en a pas moins constitué pour Félicité Angers comme pour plusieurs écrivains de l'époque l'espace improbable mais privilégié de la construction d'une subjectivité indissociablement sociale et littéraire.

67 Louis Rousseau, "L'imprimé religieux et ses lecteurs au Québec», Yvan Lamonde, Patricia Fleming et Fiona A. Black (dir.), op. cit., p. 380.68 Lucie Robert, loc. cit. p. 434. 